\title{
Extended pleurectomy and decortication with resection and reconstruction of pericardium and hemidiaphragm for malignant pleural mesothelioma
}

\author{
Raphael S. Werner, Claudio Caviezel, Olivia Lauk, Isabelle Opitz \\ Department of Thoracic Surgery, University Hospital Zurich, Zurich, Switzerland \\ Contributions: (I) Conception and design: All authors; (II) Administrative support: RS Werner, O Lauk; (III) Provision of study materials or patients: \\ C Caviezel; (IV) Collection and assembly of data: RS Werner, O Lauk, C Caviezel; (V) Data analysis and interpretation: All authors; (VI) Manuscript \\ writing: All authors; (VII) Final approval of manuscript: All authors. \\ Correspondence to: Isabelle Opitz, MD, FEBTS. Senior Attending Surgeon, Department of Thoracic Surgery, University Hospital Zurich, Rämistrasse \\ 100, 8091 Zurich, Switzerland. Email: Isabelle.schmitt-opitz@usz.ch.
}

\begin{abstract}
Malignant pleural mesothelioma (MPM) is a rare tumor of the visceral and parietal pleura that is most commonly associated with occupational exposure to asbestos. Since tumor biology is both aggressive and highly heterogeneous at histopathological and molecular level, prediction of prognosis and treatment effect remains challenging. Up to date, the best outcome in primary treatment is achieved by a multimodality approach that includes chemotherapy, followed by macroscopic complete resection (MCR). However, due to the morbidity associated with radical surgery and the limited life expectancy, a deliberate allocation to MCR is crucial. While MCR can be accomplished by extrapleural pneumonectomy (EPP) or extended pleurectomy and decortication (EPD), the current preferential treatment is EPD due to its lower morbidity and mortality. The present article describes the surgical perspective of MPM treatment starting off with correct allocation to MCR by applying a multimodality prognostic score (MMPS), followed by a step-wise description of the EPD with resection and reconstruction of pericardium and hemidiaphragm. We highlight the importance of repeated intraoperative biopsies and frozen section of the chest wall, the diaphragm and the pericardium to allow an accurate intraoperative staging and to adapt the surgical procedure in case of multifocal infiltration. Additionally, the prevention of possible pitfalls associated with EPD such as esophageal obstruction by the reconstructed hemidiaphragm or prolonged hospital stay due to persisting air leak are going to be discussed.
\end{abstract}

Keywords: Malignant pleural mesothelioma (MPM); macroscopic complete resection (MCR); surgical treatment; extended pleurectomy/decortication; multimodality treatment

Received: 27 October 2019; Accepted: 21 November 2019; Published: 05 April 2020.

doi: 10.21037 /jovs.2019.11.03

View this article at: http://dx.doi.org/10.21037/jovs.2019.11.03

\section{Introduction}

Malignant pleural mesothelioma (MPM) is a rare, but yet very aggressive tumor, affecting approximately 2,700 people per year in the UK and making up for less than $1 \%$ of all new cancer cases (1). More than $80 \%$ of all MPM cases are related to occupational exposure to asbestos with a latency period between 30 to 50 years. Although there is a decrease in incidence of MPM cases since its prohibition in most European countries since the mid/end eighties, MPM is expected to become a significant problem in third world countries in the next centuries (1).

According to the literature and our own institutional experience, a multimodality approach including neoadjuvant chemotherapy with platinum and anti-folate doublet, followed by macroscopic complete resection (MCR) offers better overall and disease-free survival than chemotherapy/ surgery or no therapy alone (2-5). To date, multimodality concepts including surgery are part of the most recent 

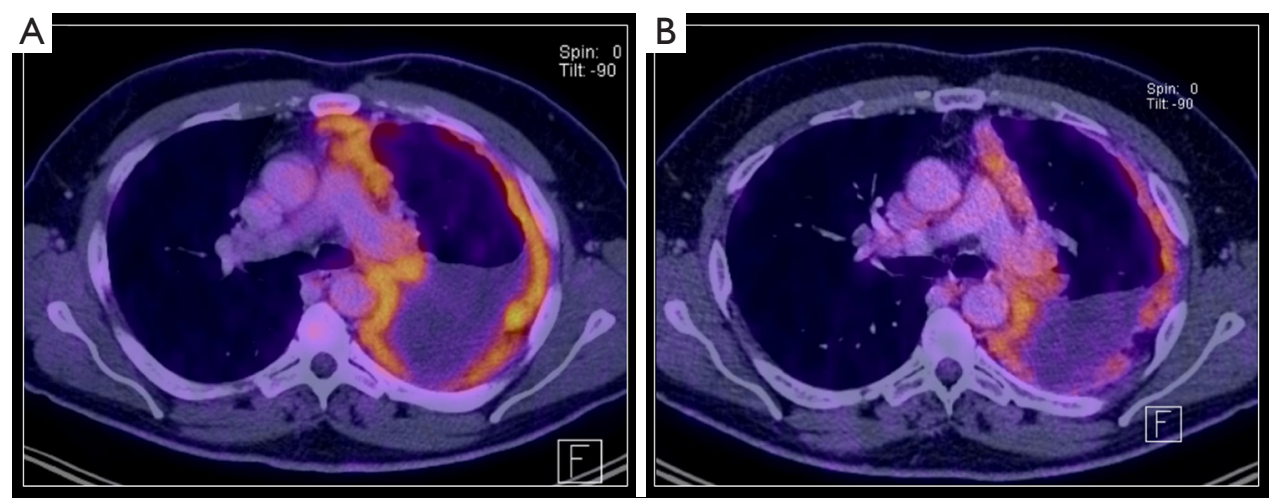

Figure 1 PET-CT imaging before and after neoadjuvant chemotherapy. (A) The PET-CT before induction chemotherapy depicts the extent of tumor infiltration and helps to identify patients with advanced tumor stages including contralateral pleural or peritoneal involvement; (B) the re-staging PET-CT is performed 2 weeks after induction chemotherapy and is assessed according to the modified RECIST criteria (14).

international guidelines $(6,7)$.

Due to the tumor's anatomical restraint, microscopic tumor will eventually be left behind. The aim of a radical surgical treatment is to achieve MCR $(5,8)$.

In the last years there has been a change in the preferred surgical approach for MPM, away from extrapleural pneumonectomy (EPP) and towards (extended) pleurectomy and decortication $[(\mathrm{E}) \mathrm{PD}](9,10)$. PD includes the resection of the visceral and parietal pleura while preserving the underlying lung. In most cases an extended PD (EPD) is performed which consists of the additional resection of the diaphragm and/or the pericardium.

In meta-analyses of several retrospective studies it was demonstrated that 30-day survival was significantly better (up to 2.5 -fold) and postoperative complications were less frequent in patients undergoing EPD compared to EPP $(3,11)$. Furthermore, the available evidence suggest that the postoperative quality of life is better after EPD (12). Nevertheless, the management of the prolonged air leak, which occurs in $90-100 \%$ of EPD cases, remains a challenge. A propensity-score matched analysis of our own data from the past 20 years showed similar rates in morbidity and comparable rates of overall survival between EPP and EPD, when treated in a multimodality therapy concept. However, 90-day mortality rates were lower for EPD than for EPP patients (0\% for EPD vs. $8 \%$ for EPP, $\mathrm{P}=0.3$ ) and the postoperative stay on the intensive care unit was significantly longer in patients undergoing EPP $\{3[1-21]$ vs. $2(0-19)$ days, $\mathrm{P}=0.001\}$ (13). In consideration of this increasing evidence, there is a tendency towards EPD in many centers in the past decade (9).

\section{Patient selection and workup}

The patient's individual treatment plan needs to be determined at an interdisciplinary tumor board with participating experts in thoracic surgery, medical oncology, radiation oncology, pneumology, radiology and pathology (6). After induction chemotherapy, a re-staging PET-CT is required prior to surgery (Figure 1). A good performance status and sufficient functional reserve to perform daily activities are essential as eligible candidates for surgery. Patient selection remains difficult, since most prognosticators are deficient and local recurrence patterns are unpredictable even for early-stages (15). However, it has been confirmed in clinical models that a number of parameters are of predictive and prognostic significance. Among those parameters, the histologic subtype is very relevant with epithelioid MPM bearing the best overall survival. Further parameters for poor survival include male sex, age $>50$ years, anemia, leukocytosis, a high serum C-reactive protein (CRP), a high serum lactate dehydrogenase (LDH), low serum albumin and a poor performance status $(16,17)$. Furthermore, several studies showed that the tumor volume at the initial diagnosis may predict the outcome after surgery and may correlate with the $\mathrm{pT} / \mathrm{pN}$ stage (18-20). At our institution, a multimodality prognostic score (MMPS) was established to use as independent prognosticator for a better patient selection. This MMP-Score was validated in other centers and enables the identification of patients who are likely to not benefit from surgery in a multimodality approach (21). The MMP-score includes four variables that are routinely assessed preoperatively: 


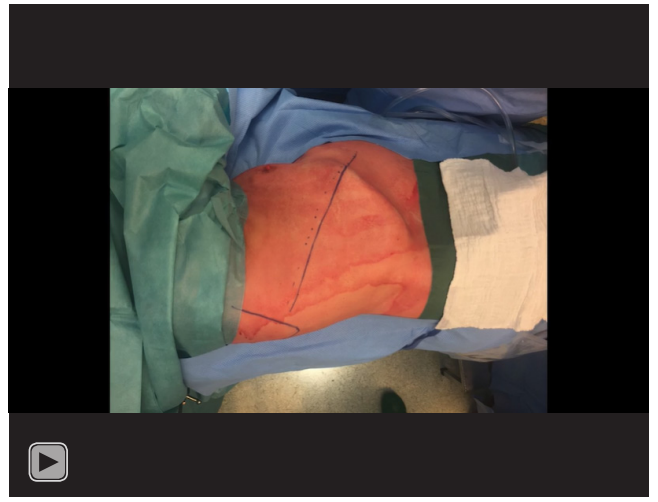

Video 1 Extended lateral thoracotomy (26).

* Tumor volume before chemotherapy $>500 \mathrm{~cm}^{3}$;

* Non-epithelioid histological subtype;

- Initial CRP-value $>30 \mathrm{mg} / \mathrm{L}$ (before chemotherapy);

* Progressive disease after chemotherapy [assessed in the re-staging PET-CT according to modified RECIST criteria (14)].

In patients with a MMPS of three or four, surgery with radical intent does not prolong overall survival when compared to untreated patients. In consideration of these findings, a multimodality approach including MCR is only performed in patients with a MMP-Score of two or smaller. In general surgery is planned within four to six weeks after the last cycle of induction chemotherapy to ensure a recovery phase for the patient after induction chemotherapy.

\section{Pre-operative preparation}

Prior to the operation, a full physical examination is carried out and standard laboratory tests are performed in our outpatient clinic. After induction chemotherapy, mild anemia is very common in patients presenting for radical surgery. Due to the inherent risk of increased perioperative blood loss, patients with anemia are preoperatively treated according to our protocol for patient blood management in order to minimize rates of blood transfusions. In patients with preoperative iron deficiency anemia, treatment consists of 1,000 $\mathrm{mg}$ iron-carboxymaltose intravenously and 40,000 IU erythropoietin alpha subcutaneously (22).

In all patients, regardless whether an EPP or an EPD is intended, the postoperative lung capacity is preoperatively estimated by lung function test and a ventilation/perfusion (V/Q) scan in order to estimate the possible extent of the operation up to a pneumonectomy in cases, where the tumor infiltrates the lung parenchyma with such an extent that no preservation of the underlying lung parenchyma is possible and EPP is justified. One of the reasons is that the lung does not account as functional anymore. For cardiologic risk stratification, the recalibrated thoracic revised cardiac risk index (RCRI) is applied (23). In patients at risk for cardiovascular disease, further examinations are performed according to the ESC/ESA guidelines on non-cardiac surgery (24). A chest magnetic resonance imaging (MRI) can help to identify tumor infiltration into surrounding structures especially in the chest wall (endothoracic fascia, intercostal musculature or ribs), diaphragm or pericardium (25).

\section{Equipment}

* Rib retractor

* Spatula for decortication

* Gore-Tex ${ }^{\circledR}$ Dualmesh $^{\circledR}$ biomaterial patch $(2 \mathrm{~mm})$ for the reconstruction of the hemidiaphragm (W.L. Gore \& Associates Inc., Flagstaff, AZ, USA)

* Acellular, biological patch for pericardial reconstruction (PeriGuard $^{\circledR}$, Lamed, Germany)

* Pleur $\mathrm{X}^{\circledR}$ drainage system for home use.

\section{Procedure}

Apart from the lateral thoracotomy, the first steps of the parietal pleurectomy are performed in doublelung ventilation to facilitate the extrapleural separation of the parietal pleura. Isolation of the affected lung is achieved thereafter via the double-lumen endotracheal tube. Intravenous antibiotic prophylaxis with amoxicillin/ clavulanic acid is administered at least 30 minutes prior to surgery. The patient is placed in lateral position and the table is buckled underneath the thorax in order to open the intercostal space.

\section{Extended lateral thoracotomy (Video 1)}

A skin incision on the level of the $6^{\text {th }}$ rib for an extended lateral thoracotomy is performed. The latissimus muscle is mobilized anteriorly and if possible dorsally retracted without cutting it. The serratus anterior muscle is divided above the $6^{\text {th }}$ rib. After incision of the periosteum, a periosteal elevator helps to shift it off the inferior angle of the $6^{\text {th }}$ rib. The $6^{\text {th }}$ intercostal space is then entered extrapleurally and a ventral excision of the costal arch is 


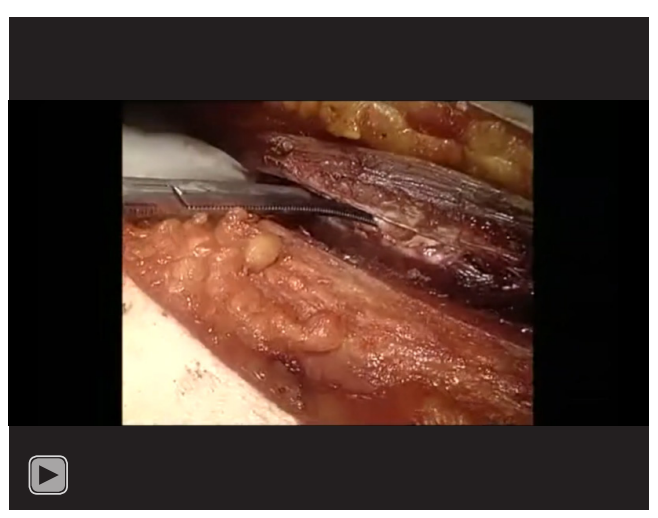

Video 2 Dissection of the parietal pleura, resection of the hemidiaphragm and the pericardium (27).

performed. This maneuver enables an extended exposure of the situs, making it possible to perform the whole operation without an additional utility thoracotomy. Furthermore, the partial chondrectomy facilitates the intercostal closure at the end of the operation. Routinely former trocar sites are excised through all layers of the chest wall and removed in a single piece.

\section{Dissection of the parietal pleura (Video 2)}

Using blunt force with swab sticks or the finger tips, the membrane between the thickened parietal pleura and the endothoracic fascia is first dissected in ventral direction and towards the apex.

During the procedure, biopsies of the pericardium and the chest wall, as well as any lesion with suspicion of infiltrating character should be taken for frozen section. In case of microscopic infiltration, further resection is required if feasible. In cases where multilevel chest wall infiltration is proven, a partial pleurectomy without decortication of the visceral pleura is a reasonable alternative to EPD in our opinion. In anterior and apical direction, the internal mammary artery and the subclavian vessels need to be located and preserved. For secure hemostasis, we recommend sharp preparation and double-clipping of the side branches of the internal mammarian vessels. The dissection is further proceeded towards the mediastinum. While on the right side, the superior vena cava and the azygous vein need to be approached with caution, on the left side, precaution is warranted for the aortic arch its vessels and the descending aorta. If possible, the phrenic nerve and, in particular, the recurrent laryngeal nerve should

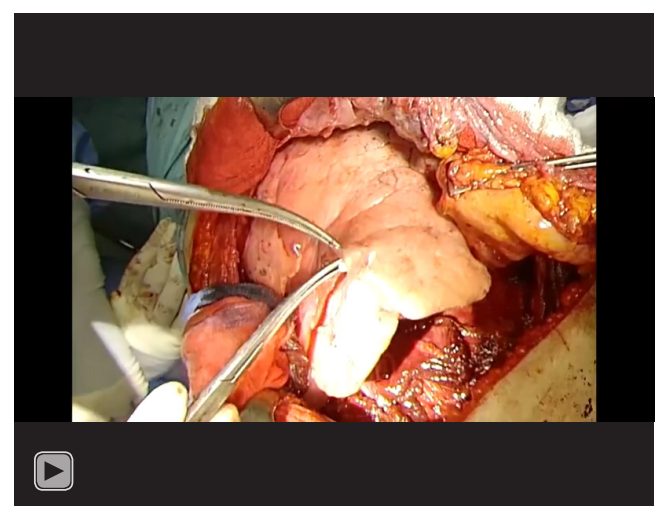

Video 3 Decortication of the visceral pleura (28).

be preserved, especially in patients with low tumor load and the possibility to preserve the diaphragm. The patient will profit postoperatively due to increased respiratory function. After dissection of the hilar structures, the pleura is separated from the esophagus. A large nasogastric tube should routinely be placed and helps to palpate the esophagus and distinguish it from surrounding tissue. If macroscopic or microscopic (confirmed by frozen section) infiltration of the pericardium is present, the pericardium is resected as well.

Subsequently, the costophrenic sinus is dissected and the parietal pleura is removed from the diaphragm. In case of macroscopic or microscopic infiltration, resection of the diaphragm is indicated. By using swab sticks, the diaphragm is retracted and dissected from the peritoneum. If peritoneal lesions become apparent, biopsies should be taken for frozen section and the peritoneal cavity should be endoscopically visualized in order to exclude further peritoneal dissemination.

\section{Decortication of the visceral pleura (Video 3)}

After pleurectomy of the parietal pleura, the pleural cap encasing the lung is opened and an incision is made superficially into the visceral pleura of each lobe. Decortication is performed on a ventilated ipsilateral lung with a positive end-expiratory pressure (PEEP) of $5-10 \mathrm{mmHg}$. Starting from the incision, the visceral pleura is dissected from the lung parenchyma via blunt dissection or with small swab sticks. The complete removal of any anthracotic pigment on the lung surface helps to identify and confirm that the right layer of visceral pleura is being 


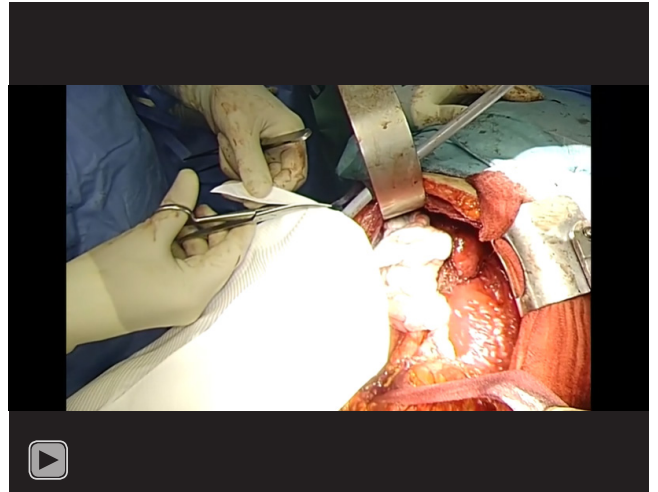

Video 4 Reconstruction of the pericardium and the hemidiaphragm (29).

dissected. The visceral pleura is peeled off towards the apex and the basis of every lobe, followed by interlobar dissection towards the hilus. Especially the dissection along the fissures is challenging. If possible, the tumor tissue is removed in a single piece containing visceral and parietal pleura, as well as the resected diaphragm and pericardium. In case of infiltration of the lung parenchyma, single wedgeresections might be necessary. The decortication of the lobes' atelectatic edges can be similarly challenging and also here, wedge-resections can facilitate the procedure.

\section{Reconstruction of the pericardium and the hemidiaphragm (Video 4)}

For reconstruction of the diaphragm, a Gore-Tex ${ }^{\circledR}$ Dualmesh ${ }^{\circledR}$ biomaterial patch (W.L. Gore \& Associates Inc., Flagstaff, AZ, USA) is put in place and fixed at the chest wall with a 1-0 Prolene ${ }^{\circledR}$ suture loop. The Gore-Tex ${ }^{\circledR}$ Patch is oriented with the rough CORDUROY ${ }^{\circledR}$ surface facing the lung to enhance adhesions and minimize residual pleural space. Sutures should be placed around the adjacent ribs to provide additional fixation. In order to compensate for the smaller residual lung after decortication, we attach the patch almost horizontally and more cranial than the diaphragm's original position and fix it directly around the rib below the thoracotomy. By minimizing the pleural space, it is intended to reduce the subsequent seropneumothorax and the coherent risk of pleural infections. On the left side, a gap as large as about a fingertip needs to be left towards the esophagus to avoid subsequent anatomical or functional obstruction by the Gore-Tex ${ }^{\circledR}$ patch and to avoid gastric herniation. Towards the entry of the inferior vena cava and the esophagus, additional point sutures are used to attach the Gore-Tex ${ }^{\circledast}$ patch.

The resected pericardium is reconstructed with an acellular biological patch. In order to avoid cardiac herniation and to allow an evacuation of accumulating fluid, the pericardial sac should be loosely closed and a small gap should be left open towards its top.

\section{Lymphadenectomy}

We recommend systematic lymphadenectomy of the mediastinal lymph nodes according to lung cancer surgery (right side: ATS 2R, 4R, 7, 10R; left side: ATS 5, 6, 7, $10 \mathrm{~L})$ and the internal mammary and intercostal nodes if applicable.

\section{Hemostasis and closure of the thoracotomy}

After obtaining hemostasis by argon beaming and irrigation with povidone-iodine diluted saline solution, three chest tubes should be placed in anteroapical, posteroapical and basal direction. At our center, a Pleur $\mathrm{X}^{\circledR}$ drainage system (Becton Dickinson, Franklin Lakes, NJ, USA) is used instead of a third chest tube in cases where visceral decortication was performed. With a high incidence of prolonged air-leak in these patients, this indwelling catheter system may reduce the duration of hospital stay by enabling easy and safe home-usage. The thoracotomy is closed with a loop suture, followed by re-adaptation of the serratus anterior muscle, subcutaneous and intracutaneous suture.

\section{Tips, tricks and pitfalls}

* High intraoperative $\mathrm{FiO}_{2}$ and/or barotrauma after induction chemotherapy can trigger an acute respiratory distress syndrome (ARDS). It has been shown in previous analyses, that centers with a low volume of EPPs have a significantly higher incidence in ARDS. Therefore, the center's EPD case volume and its capability to handle a potential ARDS by offering support through extracorporal membrane oxygenation (ECMO) significantly influences morbidity and mortality in MPM surgery (30).

* During the dissection of the parietal pleura, biopsies of the endothoracic fascia and its underlying tissue, the diaphragm and the pericardium should be taken. Frozen section is used to exclude multilevel chest wall infiltration. In case of multifocal chest wall infiltrations, 
an isolated parietal pleurectomy without decortication of the visceral pleura is in our opinion a reasonable alternative to a complete macroscopic resection.

* During the resection of the right hemidiaphragm and during patch reconstruction, careful dissection around the inferior vena cava is important at the diaphragm's caval hiatus.

* Due to the extent of the tumor, the esophagus can be difficult to identify. Therefore, we generally recommend to place a large nasogastric tube that helps to palpate the esophagus and distinguish it from the surrounding tissue.

* In patients with multifocal tumor infiltration in the lung parenchyma with severely compromised perfusion (V/Q-scan) of the diseased lung, single wedge resections may not be sufficient in order to achieve MCR. In such cases, an intraoperative strategical switch to EPP may be justified, of course given that the remaining lung offers sufficient quality and functional reserve.

* We recommend the reconstruction of the resected diaphragm in order to prevent gastric herniation. Since the Gore-Tex ${ }^{\circledR}$ Dualmesh $^{\circledR}$ patch is inelastic, increased tension on the patch results in increased tension on its anchorage. On the left side, a small gap sizing about a fingertip between the anchoring suture and the esophagus may avoid shear force and subsequent anatomical or functional obstruction of the esophagus.

* While the textured CORDUROY ${ }^{\circledR}$ surface of the Gore-Tex ${ }^{\circledR}$ Dualmesh ${ }^{\circledR}$ patch encourages host tissue incorporation, the other surface prevents tissue attachment. In order to minimize the residual pleural space by increasing the decorticated lung's adhesion to the neo-diaphragm, we place the CORDUROY surface facing the lung parenchyma.

* Due to the high rates of prolonged air-leak (in up to $90-100 \%$ of all patients), insertion of a Pleur $X^{\circledR}$ drainage system instead of the third chest tube can be considered. This procedure enables a simple and safe home-usage to deal with the air leak and may reduce the duration of hospital stay.

\section{Conclusions}

Current guidelines recommend a multimodality treatment including induction chemotherapy followed by MCR for a selected group of patients. At our center we overlook more than 20 years of experience with a multimodality approach including MCR. The precise patient selection within an interdisciplinary team is essential to offer an optimal treatment plan. Due to lower mortality and morbidity rates presuming a better quality of life postoperatively, EPD should be the preferred surgical approach if feasible.

Intraoperative biopsies of suspicious infiltrations of the chest wall, the diaphragm and the pericardium are essential to determine the extent of resection. In case of an intraoperatively confirmed multifocal chest wall infiltration, a partial pleurectomy without decortication of the visceral pleura is a reasonable alternative to EPD.

\section{Acknowledgments}

Funding: None.

\section{Footnote}

Conflicts of Interest: All authors have completed the ICMJE uniform disclosure form (available at https://jovs. amegroups.com/article/view/10.21037/jovs.2019.11.03/ coif). The authors have no conflicts of interest to declare.

Ethical Statement: The authors are accountable for all aspects of the work in ensuring that questions related to the accuracy or integrity of any part of the work are appropriately investigated and resolved. All procedures performed in this study were in accordance with the Helsinki Declaration (as revised in 2013). The manuscript is waived from patient informed consent according to the ethics committee or institutional review board.

Open Access Statement: This is an Open Access article distributed in accordance with the Creative Commons Attribution-NonCommercial-NoDerivs 4.0 International License (CC BY-NC-ND 4.0), which permits the noncommercial replication and distribution of the article with the strict proviso that no changes or edits are made and the original work is properly cited (including links to both the formal publication through the relevant DOI and the license). See: https://creativecommons.org/licenses/by-nc-nd/4.0/.

\section{References}

1. Delgermaa V, Takahashi K, Park EK, et al. Global mesothelioma deaths reported to the World Health Organization between 1994 and 2008. Bull World Health Organ 2011;89:716-24, 724A-724C.

2. Cao C, Tian D, Manganas C, et al. Systematic review of 
trimodality therapy for patients with malignant pleural mesothelioma. Ann Cardiothorac Surg 2012;1:428-37.

3. Cao C, Tian D, Park J, et al. A systematic review and meta-analysis of surgical treatments for malignant pleural mesothelioma. Lung Cancer 2014;83:240-5.

4. Kucukoner M, Ali Kaplan M, Inal A, et al. Clinical characteristics, treatment and survival outcomes in malignant pleural mesothelioma: an institutional experience in Turkey. J BUON 2014;19:164-70.

5. Rusch V, Baldini EH, Bueno R, et al. The role of surgical cytoreduction in the treatment of malignant pleural mesothelioma: meeting summary of the International Mesothelioma Interest Group Congress, September 11-14, 2012, Boston, Mass. J Thorac Cardiovasc Surg 2013;145:909-10.

6. Baas P, Fennell D, Kerr KM, et al. Malignant pleural mesothelioma: ESMO Clinical Practice Guidelines for diagnosis, treatment and follow-up. Ann Oncol 2015;26 Suppl 5:v31-39.

7. Kindler HL, Ismaila N, Armato SG, et al. Treatment of Malignant Pleural Mesothelioma: American Society of Clinical Oncology Clinical Practice Guideline. J Clin Oncol 2018;36:1343-73.

8. Opitz I, Weder W. Pleural mesothelioma: is the surgeon still there? Ann Oncol 2018;29:1710-7.

9. Bueno R, Opitz I, Taskforce IM. Surgery in Malignant Pleural Mesothelioma. J Thorac Oncol 2018;13:1638-54.

10. Batirel HF. Extrapleural pneumonectomy (EPP). Ann Transl Med 2017;5:232.

11. Taioli E, Wolf AS, Flores RM. Meta-analysis of survival after pleurectomy decortication versus extrapleural pneumonectomy in mesothelioma. Ann Thorac Surg 2015;99:472-80.

12. Schwartz RM, Watson A, Wolf A, et al. The impact of surgical approach on quality of life for pleural malignant mesothelioma. Ann Transl Med 2017;5:230.

13. Kostron A, Friess M, Inci I, et al. Propensity matched comparison of extrapleural pneumonectomy and pleurectomy/decortication for mesothelioma patients. Interact Cardiovasc Thorac Surg 2017;24:740-6.

14. Eisenhauer EA, Therasse P, Bogaerts J, et al. New response evaluation criteria in solid tumours: revised RECIST guideline (version 1.1). Eur J Cancer 2009;45:228-47.

15. Frauenfelder T, Kestenholz P, Hunziker R, et al. Use of computed tomography and positron emission tomography/ computed tomography for staging of local extent in patients with malignant pleural mesothelioma. J Comput Assist Tomogr 2015;39:160-5.
16. Davidson B. Prognostic factors in malignant pleural mesothelioma. Hum Pathol 2015;46:789-804.

17. Edwards JG, Abrams KR, Leverment JN, et al. Prognostic factors for malignant mesothelioma in 142 patients: validation of CALGB and EORTC prognostic scoring systems. Thorax.2000;55:731-5.

18. Frauenfelder T, Tutic M, Weder W, et al. Volumetry: an alternative to assess therapy response for malignant pleural mesothelioma? Eur Respir J 2011;38:162-8.

19. Gill RR, Richards WG, Yeap BY, et al. Epithelial malignant pleural mesothelioma after extrapleural pneumonectomy: stratification of survival with CT-derived tumor volume. AJR Am J Roentgenol 2012;198:359-63.

20. Rusch VW, Gill R, Mitchell A, et al. A Multicenter Study of Volumetric Computed Tomography for Staging Malignant Pleural Mesothelioma. Ann Thorac Surg 2016;102:1059-66.

21. Opitz I, Friess M, Kestenholz P, et al. A New Prognostic Score Supporting Treatment Allocation for Multimodality Therapy for Malignant Pleural Mesothelioma: A Review of 12 Years' Experience. J Thorac Oncol.2015;10:1634-41.

22. Muñoz M, Acheson AG, Auerbach M, et al. International consensus statement on the peri-operative management of anaemia and iron deficiency. Anaesthesia 2017;72:233-47.

23. Brunelli A, Varela G, Salati M, et al. Recalibration of the revised cardiac risk index in lung resection candidates. Ann Thorac Surg 2010;90:199-203.

24. American Society of Anesthesiologists Task Force on Perioperative Blood Management. Practice guidelines for perioperative blood management: an updated report by the American Society of Anesthesiologists Task Force on Perioperative Blood Management*. Anesthesiology 2015;122:241-75.

25. Heelan RT, Rusch VW, Begg CB, et al. Staging of malignant pleural mesothelioma: comparison of CT and MR imaging. AJR Am J Roentgenol. 1999;172:1039-47.

26. Werner RS, Caviezel C, Lauk O, et al. Extended lateral thoracotomy. Asvide 2020;7:038. Available online: http:// www.asvide.com/watch/33079

27. Werner RS, Caviezel C, Lauk O, et al. Dissection of the parietal pleura, resection of the hemidiaphragm and the pericardium. Asvide 2020;7:039. Available online: http:// www.asvide.com/watch/33080

28. Werner RS, Caviezel C, Lauk O, et al. Decortication of the visceral pleura. Asvide 2020;7:040. Available online: http://www.asvide.com/watch/33081

29. Werner RS, Caviezel C, Lauk O, et al. Reconstruction of the pericardium and the hemidiaphragm. Asvide 
2020;7:041. Available online: http://www.asvide.com/ watch/33082

30. Burt BM, Cameron RB, Mollberg NM, et al. Malignant pleural mesothelioma and the Society of Thoracic Surgeons Database: an analysis of surgical morbidity and mortality. J Thorac Cardiovasc Surg 2014;148:30-5.

doi: 10.21037 /jovs.2019.11.03

Cite this article as: Werner RS, Caviezel C, Lauk O, Opitz I. Extended pleurectomy and decortication with resection and reconstruction of pericardium and hemidiaphragm for malignant pleural mesothelioma. J Vis Surg 2020;6:20. 